\title{
Medidas para prevenção de lesão por pressão associada à posição prona durante a pandemia de COVID-19: Revisão integrativa da literatura
}

\author{
Measures for prevention of pressure injury associated with the prone position during the COVID- \\ 19 pandemic: Integrative literature review \\ Medidas para la prevención de lesiones por presión asociadas con la posición prona durante la \\ pandemia del COVID-19: Revisión integrativa de la literatura
}

Recebido: 29/03/2021 | Revisado: 09/04/2021 | Aceito: 07/05/2021 | Publicado: 21/05/2021

\author{
Wagner Luiz Da Silva Monteiro \\ ORCID: https://orcid.org/0000-0003-0418-1913 \\ Universidade Estácio de Sá, Brasil \\ E-mail: wlsmonteiro31@gmail.com \\ Natália Soares de Castro \\ ORCID: https://orcid.org/0000-0002-7992-4286 \\ Universidade Federal Fluminense, Brasil \\ E-mail: nataliasoaresdecastro@gmail.com \\ Priscila Soares de Oliveira \\ ORCID: https://orcid.org/0000-0003-2371-5079 \\ Universidade Iguaçu, Brasil \\ E-mail: crekrio@yahoo.com.br \\ Natalia da Palma Sobrinho \\ ORCID: https://orcid.org/0000-0003-3187-2832 \\ Universidade Federal do Rio de Janeiro, Brasil \\ E-mail: nataliapsobrinho@gmail.com \\ Gicélia Lombardo Pereira \\ ORCID: https://orcid.org/0000-0002-4032-2093 \\ Universidade Federal do Estado do Rio de Janeiro, Brasil \\ E-mail: gicelia.pereira@unirio.br
}

\begin{abstract}
Resumo
Objetivos: Identificar as evidências científicas acerca das medidas de prevenção de Lesão Por Pressão (LPP) associada à posição prona durante a pandemia de COVID-19. Refletir acerca das medidas de prevenção de Lesão Por Pressão associada à posição prona durante a Pandemia por COVID-19 à luz da literatura científica. Método: Revisão integrativa de literatura, utilizando os descritores "Lesão Por Pressão" e "pronação"; os critérios de inclusão foram publicações entre 2019 e 2021; em português e inglês, indexados nas bases de dados BDENF, LILACS e MEDLINE; que abordassem o tema relacionado a Lesão por Pressão em pacientes submetidos à posição prona durante a pandemia. Resultados: Análise de 5 estudos que abordavam contextualização para sustentar os objetivos propostos no estudo. As principais recomendações preventivas para lesão por pressão em pacientes submetidos a posição prona foram: avaliação diária da pele com identificação de riscos, controle de umidade, redistribuição de pressão com uso de coxins, hidratação da pele, rotação da cabeça a cada duas horas e o uso de coberturas preventivas. Conclusão: Dentre os achados resultantes desta pesquisa nos chamou a atenção o uso da telemedicina, consulta virtual, para auxílio na prevenção de feridas, os cuidados com a pele elencados com ênfase na solução com PH entre 4,0 e 7,0, o uso de coberturas preventivas, uso de superfícies para redistribuição de pressão, conhecimento dos pontos predispostos ao desenvolvimento de lesões por pressão devem deter atenção especial às medidas protetivas, profissionais especializados no manejo deste perfil de pacientes e a necessidade de sanar os fatores intrínsecos relacionados.
\end{abstract}

Palavras-chave: Infecção por coronavírus; Decúbito ventral; Prona; Lesão por pressão.

\begin{abstract}
Objectives: To identify the scientific evidence about pressure injury prevention (LPP) measures associated with the prone position during the COVID-19 pandemic. Reflect on the Pressure Injury prevention measures associated with the prone position during the COVID-19 Pandemic in the light of the scientific literature. Method: Integrative literature review, using the keywords "Pressure Injury" and "pronation"; the inclusion criteria were publications between 2019 and 2021; in Portuguese and English, indexed in the databases BDENF, LILACS and MEDLINE; that addressed the issue related to Pressure Injury in patients submitted to the prone position during the pandemic. Results: Analysis 5 studies that addressed contextualization to support the objectives proposed in the study. The main preventive
\end{abstract}


recommendations for pressure injuries in patients submitted to the prone position were: daily skin assessment with risk identification, humidity control, pressure redistribution with the use of cushions, skin hydration, head rotation every two hours and the use of preventive coverings. Conclusion: Among the findings resulting from this research, the use of telemedicine, virtual consultation, to assist in wound prevention, skin care listed with emphasis on the solution with a $\mathrm{pH}$ between 4.0 and 7.0, the use of of preventive coverings, use of surfaces for redistribution of pressure, knowledge of the points predisposed to the development of pressure injuries must pay special attention to protective measures, professionals specialized in the management of this patient profile and the need to remedy the intrinsic factors related.

Keywords: Coronavirus infection; Prone position; Prone; Pressure injury.

\section{Resumen}

Objetivos: Identificar la evidencia científica sobre las medidas de prevención de lesiones por presión (LPP) asociadas con la posición prona durante la pandemia de COVID-19. Reflexione sobre las medidas de prevención de lesiones por presión asociadas con la posición boca abajo durante la pandemia COVID-19 a la luz de la literatura científica. Método: Revisión integrativa de la literatura, utilizando las palabras clave "Lesión por presión" y "pronación"; los criterios de inclusión fueron publicaciones entre 2019 y 2021; en portugués e inglés, indexado en las bases de datos BDENF, LILACS y MEDLINE; que abordó el problema relacionado con las lesiones por presión en pacientes sometidos a la posición de decúbito prono durante la pandemia. Resultados: Análisis de 5 estudios que abordaron la contextualización para apoyar los objetivos propuestos en el estudio. Las principales recomendaciones preventivas de las lesiones por presión en los pacientes sometidos a decúbito prono fueron: valoración cutánea diaria con identificación de riesgos, control de la humedad, redistribución de la presión con el uso de cojines, hidratación de la piel, rotación de la cabeza cada dos horas y uso de cubiertas preventivas. Conclusión: Entre los hallazgos resultantes de esta investigación, el uso de la telemedicina, la consulta virtual, para ayudar en la prevención de heridas, el cuidado de la piel enumerado con énfasis en la solución con un $\mathrm{pH}$ entre 4.0 y 7.0, el uso de recubrimientos preventivos, el uso de superficies para La redistribución de la presión, el conocimiento de los puntos predispuestos al desarrollo de lesiones por presión deben prestar especial atención a las medidas de protección, los profesionales especializados en el manejo de este perfil de paciente y la necesidad de remediar los factores intrínsecos relacionados.

Palabras clave: Infección por coronavirus; Posición prona; Prona; Lesión por presión.

\section{Introdução}

Originado na cidade de Wuhan, na China, o contágio pelo Coronavírus a humanos ocorreu pela contaminação de animais, possivelmente, em um mercado público local. O SARS-CoV-2 pertence a uma larga espécie de vírus comumente encontrada em animais selvagens ou não, como gatos, morcegos, gado e camelos. Ao sofrer mutação este vírus se tornou apto a infectar humanos e desenvolver até mesmo, em alguns casos, a Síndrome Respiratória Aguda Grave (SARS) (Brasil, 2020).

Identificados desde 1960, os Coronavírus são microorganismos de material genético constituído por RNA, e causam acometimento respiratório de leve a grave em seres humanos. Dentre esta família de vírus, advindo de morcegos para humanos, encontram-se os SARS-CoV, causador da Síndrome Respiratória Aguda Grave ou SARS; os MERS-CoV, causador da Síndrome Respiratória do Oriente Médio ou MERS e atualmente o nCoV-2019, o novo tipo de Coronavírus (Durigon, 2020).

Com a pandemia pelo COVID-19 e as complicações associadas, aumentou-se a demanda pelo uso de ventilação mecânica (VM) em função do acometimento pulmonar pela Insuficiência Respiratória, Hipoxemia e a Síndrome do Desconforto Respiratório Agudo (SDRA), infligido a este paciente. Como medida adjuvante, a otimização desta terapêutica fez-se o uso da posição prona, a fim de melhorar a resposta e a complacência pulmonar, aumentando a oxigenação tecidual. Porém, tal terapêutica apresenta riscos pertinentes ao desenvolvimento de Lesões Por Pressão (LPP), implicando em maiores danos ao paciente (Sanduzzi, 2020).

Lesão Por Pressão (LPP) é a terminologia adotada em 2016 pela National Pressure Ulcer Advisory Panel, utilizada para indicar uma lesão localizada na pele e em outros tecidos adjacentes, como tecido muscular, fáscia muscular, tendão e proeminência óssea, que é decorrente da junção de pressão, cisalhamento e fricção, sendo que um dos fatores que contribuem para a abertura dessas lesões.São os fatores relacionados para as mesmas: à nutrição, diminuição da mobilidade e diminuição da percepção sensorial, idade, sexo, fatores fisiopatológicos, dentre outros. Todos esses fatores podem interferir na redução de fluxo sanguíneo, alterando a funcionalidade celular dos tecidos expostos à pressão. (Moraes, 2016). 
O mecanismo de desenvolvimento de uma lesão tecidual se inicia quando os artifícios celulares de adaptação ao dano incidido falham, ocasionando dano reversível ou não ao tecido. A pressão na extremidade dos vasos ocasiona seu colabamento, e assim a cessação do aporte de nutrientes e oxigênio ao tecido, culminando no sofrimento celular e possível evolução para morte. Diversos fatores influenciam no processo de morte celular em LPP, sejam eles: o tipo de célula envolvida, o nível e tempo de pressão aplicada, a ocorrência de fricção e cisalhamento, a umidade e temperatura do tecido, as condições nutricionais, a idade do indivíduo, quadro clínico e, dentre outros, como uso de alguns medicamentos (Campos, Souza, Vasconcelos, Lucena \& Assis Gomes. 2016).

Nesse sentido, uma complicação potencial da pronação são as Lesões Por Pressão, que se desenvolvem em regiões anatômicas diferentes daquelas tipicamente vistas em pacientes acamados em decúbito dorsal. O envolvimento nas regiões malar, nasal e frontal, bem como na mandíbula, lábios, região esternal e cristas ilíacas não é incomum, pois esses pacientes são hemodinamicamente lábeis e instáveis, e permanecem em uma posição fixa por até 20 horas na posição prona. Os riscos adicionais incluem edema facial, instabilidade hemodinâmica transitória, abrasões da córnea e obstrução do tubo endotraqueal (NPIAP, 2020).

O decúbito ventral também conhecido atualmente como posição prona ou pronação. Consiste no posicionamento do paciente com o abdome voltado para a superfície da cama e a cabeça lateralizada. Descrito pela primeira vez por em 1974 sugerindo que pacientes submetidos a esta posição poderiam apresentar melhor expansão pulmonar na região dorsal, o estudo foi confirmado posteriormente com a posição prona refletindo em melhora da pressão parcial de oxigênio. $O$ decúbito ventral é associado a benefícios na ventilação e oxigenação de pacientes acometidos por patologias pulmonares por aperfeiçoar o fluxo de ar em áreas anteriormente colabadas (Morsch, 2018).

Atualmente sabemos a importância do Enfermeiro na linha de frente ao cuidado com o paciente na tomada de decisões, no gerenciamento de pessoas, e no manuseio e habilidades que se renovam a cada dia. Esse cuidado se torna diferenciado e científico quando é desenvolvido a partir de práticas baseada em evidência, utilizando pesquisas de práticas clínicas, tendo como um dos recursos a revisão de literatura (Galvão \& Ursi, 2016).

A idealização deste estudo surge durante a atividade assistencial na modalidade de treinamento em serviço do curso de Pós-Graduação em Enfermagem nos moldes de Residência, a partir do acompanhamento do uso da posição prona com maior frequência em paciente acometidos pela COVID-19 e a preocupação da equipe no tocante ao desenvolvimento de LPP. Tal prática não fazia parte da rotina da equipe de Enfermagem o que propiciou a reflexão quanto à necessidade por conhecimento pertinente a técnica postural e sua relação com LPP, para assim promover um cuidado seguro e livre de danos.

Dessa forma, diante do atual cenário pandêmico é possível perceber o papel substancial do Enfermeiro na linha de frente do cuidado ao paciente acometido pela COVID-19 em pronação, e com isso esse estudo propõe como objeto as medidas de prevenção de Lesão Por Pressão relacionada a posição prona, com a formulação da seguinte questão de pesquisa: "Quais evidências científicas disponíveis acerca das medidas para prevenção de Lesão Por Pressão associada à posição prona durante a pandemia do Covid-19?", e com o intuito de atingir o objeto do estudo, elaboramos os seguintes objetivos: Identificar as evidências científicas acerca das medidas de prevenção de Lesão Por Pressão associada à posição prona durante a Pandemia de COVID-19 e refletir acerca das medidas de prevenção de Lesão Por Pressão associada à posição prona durante a pandemia por COVID-19 à luz da literatura científica.

A problemática do estudo justifica-se por estar alicerçada com a meta seis do Programa Nacional de Segurança do Paciente que trata sobre a recomendação de medidas de enfrentamento para a prevenção de ocorrência de LPP, corrobora com resolução COFEN n 639/2020 que dispõe sobre a participação do Enfermeiro no cuidado aos pacientes em Ventilação Mecânica e por tratar-se de um fenômeno biológico novo de magnitude mundial, que precisa de subsídios técnicos científicos para construção de pensamento crítico social. 


\section{Metodologia}

Trata-se de uma revisão integrativa da literatura, que une estudos de diferentes metodologias, permitindo que uma análise seja realizada sem que a filiação epistemológica seja alterada (Soares, Resck, Terra \& Camelo, 2015).

A revisão integrativa foi realizada a partir da coleta de dados de fontes secundárias, através do levantamento bibliográfico nas bases de dados científicas guiado por critérios rigorosos de inclusão, questão norteadora e em experiências evidenciadas.

A revisão integrativa da literatura reúne diversas vertentes, ordenadamente, a partir de uma busca científica de determinado assunto no acervo da literatura, e com isso, são discutidas e concluídas em um conceito científico teórico para contribuição da ciência. (Botelho, Almeida \& Macedo, 2011).

Para a elaboração do estudo foram seguidos os seis passos descritos por Botelho, Almeida e Macedo (2011):

Figura 1: Etapas da revisão integrativa.

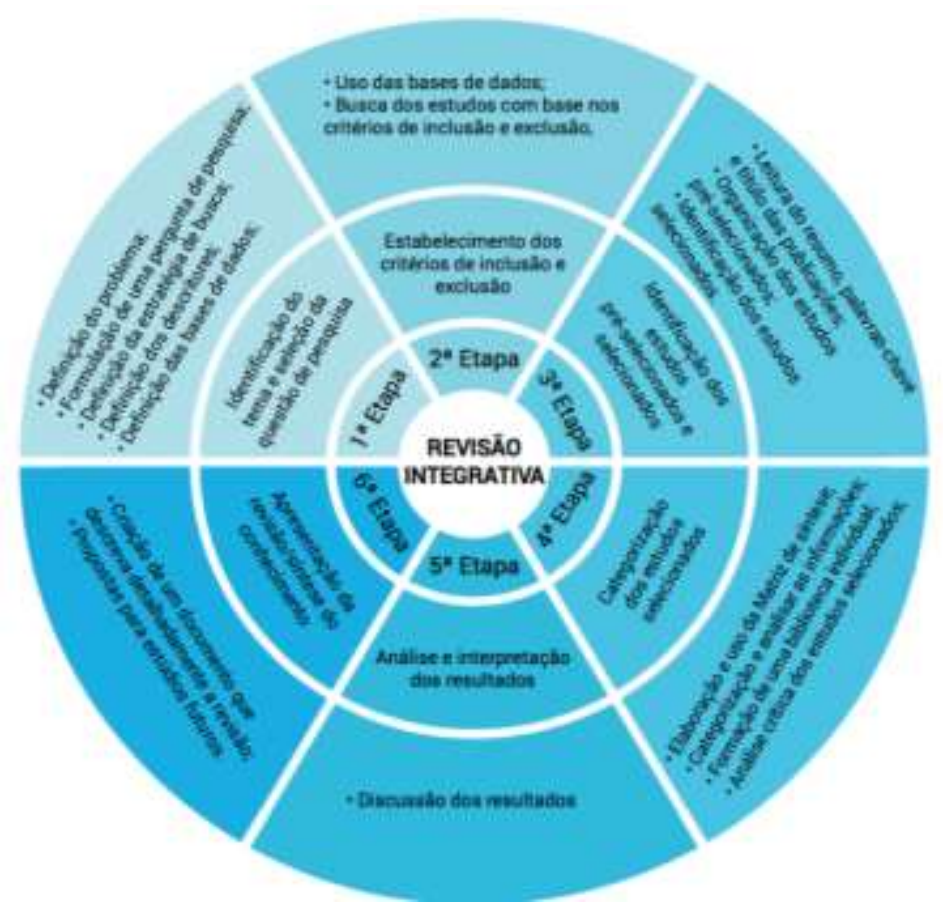

Fonte: Botelho, Almeida e Macedo (2011).

Para levantamento dos artigos, a seleção foi realizada nas seguintes bases de dados: Literatura Latino-Americana e do Caribe em Ciências da Saúde (LILACS), Banco de dados em enfermagem (BDENF) e Medical Literature Analysis and Retrieval System Online (MEDLINE) via BVS com auxílio dos seguintes descritores na linguagem em português e inglês: Infecção por coronavírus OR Decúbito Ventral OR Prone Position OR Prona AND Lesão por Pressão OR Pressure Ulcer.

A busca de dados preliminar ocorreu no período que compreendeu de dezembro de 2010 a janeiro de 2021. Foram utilizados os filtros: textos completos e disponíveis. Os critérios de inclusão foram artigos no idioma Inglês e Português e que abordassem o tema relacionado à Lesão por Pressão em pacientes submetidos à posição prona durante a pandemia com o objetivo de responder à questão norteadora.

Dessa forma, seguindo tais parâmetros, a busca nas bases de dados resultou na identificação de 51 artigos que após a exclusão de triagem de tempo e avaliação dos textos completos, foram encontrados 5 artigos (Figura 2). 
Figura 2: Fluxograma com os resultados da seleção dos artigos.
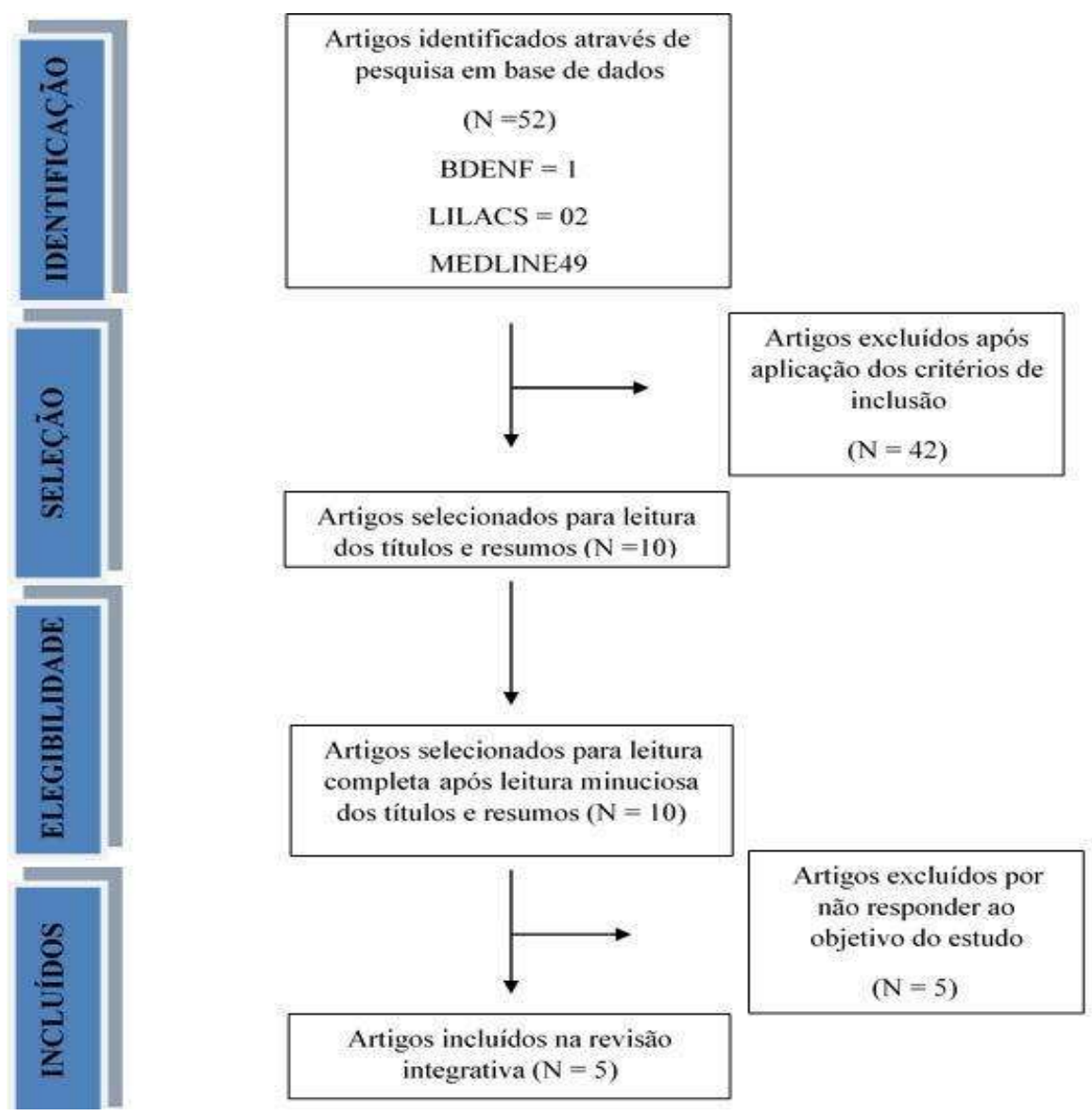

Fonte: Autores (2021).

Posteriormente, foi realizada a avaliação dos estudos incluídos na revisão integrativa, análise crítica e correlação com a questão norteadora de pesquisa. Para a análise e síntese dos artigos que atenderam aos critérios de inclusão foi utilizado um quadro sinóptico especialmente construído para esse fim, que contemplou os seguintes aspectos, considerados pertinentes: título da pesquisa; autores; metodologia; resultados; conclusões e nível de evidência.

A seguir, foi contemplado a interpretação e discussão dos resultados, destacando-se os trabalhos que trouxeram a relação com a questão norteadora e por último, foi apresentada a revisão e síntese do conhecimento produzido acerca das principais relações entre Lesão por Pressão e posicionamento prona durante a pandemia por COVID-19.

\section{Resultados}

Foram encontrados 53 artigos identificados em base de dados, sendo que somente 10 artigos estavam disponíveis em Português e Inglês, completos no intervalo dos últimos dois anos (2019-2021). A partir dos 10 artigos pré-selecionados, utilizouse o critério de inclusão e exclusão da pesquisa a fim de atender ao objetivo do objetivo do estudo. Assim, emergiu como amostra para compor este estudo cinco referências como citado na Figura 2.

O Quadro 1 traz o perfil das produções científicas acerca da prevenção de lesão por pressão associada à posição prona durante a pandemia de COVID-19 e permitem a reflexões acerca das medidas de prevenção de lesão por pressão associada à posição prona durante a pandemia por COVID-19 entre 2019 e 2021.

Observa-se ausência de artigos abordando o tema em questão no banco de dados BDEN. Não há a predominância de uma nacionalidade dentre as publicações, sendo os países: Estados Unidos da América (1), Brasil (1), Irlanda (1), França (1) e 
Itália (1). Houve um predomínio dos artigos publicados no idioma inglês. Com relação ao método, destacam-se o predomínio de relatos de caso, com quatro artigos, e uma carta ao editor conferindo assim a classificação quanto ao nível de evidência como 4 para os trabalhos de relato de caso e 5 para um trabalho classificado como carta ao editor.

O Quadro 2 apresenta a síntese das produções presentes na literatura.

Quadro 1: Perfil das produções científicas. RJ, 2021.

\begin{tabular}{|c|c|c|c|c|c|c|}
\hline $\mathbf{N}$ & $\begin{array}{l}\text { Base de } \\
\text { dados }\end{array}$ & Título & Autor & $\begin{array}{c}\text { Ano } \\
\text { Categoria }\end{array}$ & País & $\begin{array}{c}\text { Nível } \\
\text { de } \\
\text { evidência }\end{array}$ \\
\hline 1 & MEDLINE & $\begin{array}{l}\text { Medical Device-Related } \\
\text { Pressure Injuries During the } \\
\text { COVID-19 Pandemic. }\end{array}$ & Martel, Tanya; Orgill, Dennis P & $\begin{array}{c}2020 \\
\text { Enfermagem }\end{array}$ & EUA & 4 \\
\hline 2 & MEDLINE & $\begin{array}{l}\text { A multidisciplinary approach } \\
\text { to prevent and treat pressure } \\
\text { sores in proned COVID-19 } \\
\text { patients at a quaternary } \\
\text { university hospital. }\end{array}$ & $\begin{array}{l}\text { Busnardo, Fabio de Freitas; Monteiro, } \\
\text { Gustavo Gomes; Mendes, Rogério } \\
\text { Rafael da Silva; Abbas, Laielly; Pagotto, } \\
\text { Vitor Figueiredo; Camargo, Cristina; } \\
\text { Carmona, Maria José Carvalho; } \\
\text { Gemperli, Rolf. }\end{array}$ & $\begin{array}{c}2020 \\
\text { Médico } \\
\text { Enfermeiro } \\
\text { Fisioterapeuta }\end{array}$ & Brasil & 4 \\
\hline 3 & MEDLINE & $\begin{array}{l}\text { Prevention of pressure ulcers } \\
\text { among individuals cared for } \\
\text { in the prone position: lessons } \\
\text { for the COVID-19 } \\
\text { emergency. }\end{array}$ & $\begin{array}{l}\text { Zena Moore, Declan Patton, Pinar } \\
\text { Avsar,Natalie L McEvoy, Gerard } \\
\text { Curley, AgleciaBudri, Linda Nugent, } \\
\text { Simone Walsh, Tom O'Connor }\end{array}$ & $\begin{array}{c}2020 \\
\text { Enfermagem }\end{array}$ & Irlanda & 4 \\
\hline 4 & MEDLINE & $\begin{array}{c}\text { Facial pressure ulcers in } \\
\text { COVID-19 patients } \\
\text { undergoing prone } \\
\text { positioning: How to prevent } \\
\text { an underestimated epidemic? }\end{array}$ & $\begin{array}{c}\text { Perrillat, A; Foletti, JM; Lacagne, AS; } \\
\text { Guyot, L; Graillon, N }\end{array}$ & $\begin{array}{c}2020 \\
\text { Médico }\end{array}$ & França & 4 \\
\hline 5 & MEDLINE & $\begin{array}{l}\text { Pression-induced facial } \\
\text { ulcers by prone position for } \\
\text { COVID-19 mechanical } \\
\text { ventilation }\end{array}$ & $\begin{array}{l}\text { Ramondetta, Alice; Ribero, Simone; } \\
\text { Costi, Sonia; Dapavo, Paolo }\end{array}$ & $\begin{array}{c}2020 \\
\text { Médico }\end{array}$ & Itália & 5 \\
\hline
\end{tabular}

Fonte: Autores (2021). 
Quadro 2: Evidências científicas observadas nas produções. RJ, 2021

\begin{tabular}{|c|c|c|}
\hline $\mathbf{N}$ & Objetivo & Conclusões \\
\hline 1 & $\begin{array}{l}\text { Relatar a experiência obtida na busca em } \\
\text { desenvolver iniciativas para melhora na } \\
\text { qualidade dos indicadores de Lesões por pressão } \\
\text { relacionadas a dispositivos médicos. }\end{array}$ & $\begin{array}{l}\text { Identificaram-se as áreas de melhoria: compreender as necessidades de aprendizagem } \\
\text { da equipe, uso de coberturas preventivas, dispositivos de fixação endotraqueal, } \\
\text { superfícies para redistribuição de pressão e consulta virtual para ferida. }\end{array}$ \\
\hline 2 & $\begin{array}{l}\text { Relatar a experiência na tomada de decisão à } \\
\text { alta incidência de Lesões por pressão em } \\
\text { pacientes submetidos à prona. }\end{array}$ & $\begin{array}{l}\text { As medidas tomadas foram: tratamento cirúrgico, quando necessário, inspeção diária, } \\
\text { higiene, hidratação, controle da umidade e temperatura da pele, redução da pressão } \\
\text { nas proeminências ósseas com mudança na posição do corpo a cada } 2 \text { horas e } \\
\text { almofadas de proteção desenvolvidas especificamente para cada caso. }\end{array}$ \\
\hline 3 & $\begin{array}{l}\text { Identificar e revisar recomendações baseadas em } \\
\text { evidências desenvolvidas para facilitar a seleção } \\
\text { e aplicação de medidas preventivas destinadas a } \\
\text { reduzir o desenvolvimento de UP em pacientes } \\
\text { ventilado na posição prona. }\end{array}$ & $\begin{array}{l}\text { Apresenta, com base nas evidências, recomendações para facilitar a seleção e } \\
\text { aplicação de intervenções preventivas para reduzir o desenvolvimento de Lesões por } \\
\text { pressão relacionado ao posicionamento prona sendo: avaliação da pele deve ser } \\
\text { realizada antes da pronação e antes de reposicionar o paciente de volta para posição } \\
\text { supina, manter a pele limpa e hidratada, usar limpadores com pH balanceado, } \\
\text { dispositivos posicionados para descarregar a pressão nos pontos do rosto e no corpo, } \\
\text { uso regular de coberturas de proteção, como hidrocolóides, filme transparente e } \\
\text { silicone. }\end{array}$ \\
\hline 4 & $\begin{array}{l}\text { Enfatizar o risco de úlceras de pressão facial em } \\
\text { decúbito ventral, a fim de discutir sua } \\
\text { fisiopatologia e destacam a importância de ações } \\
\text { preventivas adequadas. }\end{array}$ & $\begin{array}{l}\text { As medidas de prevenção elencadas são: Treinamento da equipe de cuidados } \\
\text { intensivos, supervisão e monitorização da pronagem por enfermeiro familiarizado } \\
\text { com a técnica, uso de coxim específico para a posição prona, com descompressão do } \\
\text { masseter e espaço para o tubo orotraqueal, ou coxim a base de silicone, ou géis de } \\
\text { silicone, rotação regular da cabeça, fixação adequada do tubo orotraqueal com } \\
\text { espuma de silicone como proteção para bochechas, corrigir preventivamente } \\
\text { parâmetros sistêmicos relacionados a cicatrização como hipoxemia, anemia ou } \\
\text { desnutrição. }\end{array}$ \\
\hline 5 & $\begin{array}{l}\text { Relatar experiência no manejo de lesão facial } \\
\text { em paciente submetido a prona. }\end{array}$ & $\begin{array}{c}\text { Foram utilizados curativos avançados para tratamento da lesão desenvolvida, à base } \\
\text { de colagenase, para dissolver o componente fibrinoso e as crostas, e espuma de } \\
\text { poliuretano com função absorvente e protetora. }\end{array}$ \\
\hline
\end{tabular}

Fonte: Autores (2021).

\section{Discussão}

Martell e Orgil (2020) além de Busnardo et al. (2020) nos trazem como a pandemia pela covid 19 resultou no aumento de pacientes posicionados em posição prona aumentou rapidamente, resultando em uma incidência de oitenta por cento de desenvolvimento de lesões por pressão nestes pacientes. sobrecarregando os sistemas para prevenção de lesões por pressão, junto a esse fato o acometimento de lesões por pressão relacionadas a esta modalidade terapêutica trazendo a relação de três vezes mais em relação a posição supino.

O aumento na demanda de pacientes em posição prona, resultante da pandemia, se mostra como um fator predisponente ao desenvolvimento de lesões por pressão.

Martell e Orgil (2020) relatam que o uso de dispositivos médicos está diretamente associado ao desenvolvimento de lesões quando associados à posição prona. Como fatores que propiciam o desenvolvimento destas lesões estão, segundo os 
autores, a sedação, o aumento da umidade e pressão; ressaltam também fatores intrínsecos como o aumento da liberação de occitocinas, disfunção endotelial e isquemia, contribuindo para o desenvolvimento de lesões.

Tais observação do autor se fazem imprescindíveis à tarefa de prevenção das lesões por pressão, uma vez que a identificação adequada dos fatores relacionadas propicia o desenvolvimento de escalonamento adequado dos riscos e adoção de medidas preventivas assertivas.

Martell e Orgil (2020) também nos traz, em concordância com Busnardo et al. (2020) como medidas adotadas para prevenção o emprego de equipe multidisciplinar destinada especificamente para a pronação dos pacientes eleitos, elaboração de plano de cuidados específico, protocolo clínico com fotografias do posicionamento apropriado, descrição dos produtos a serem utilizados e educação em saúde às equipes de enfermagem. $\mathrm{O}$ uso de coberturas preventivas, dispositivos de adequada fixação endotraqueal, coxins para redistribuição de pressão e consulta virtual são apresentados como medidas efetivas para prevenção de lesão por pressão.

Busnardo et al. (2020) referem que o envolvimento de uma equipe de inovação técnica no hospital possibilitou o desenvolvimento de coxins especificamente para cada caso em parceria com a iniciativa privada, mostrando como melhor alternativa aos pacientes submetidos à prona por longos períodos.

Tal iniciativa nos traz a reflexão sobre o agregamento positivo da contribuição de profissionais não envolvidos diretamente com os cuidados à prevenção de lesões por pressão e o diferencial destes profissionais presentes no hospital do estudo debruçados sobre inovações técnicas.

Moore et al. (2020), afirmam que a avaliação diária da pele é a melhor prática preventiva, e nesse sentido, foi recomendado a realização duas vezes ao dia em pacientes propensos ao extravasamento de fluidos e acometidos de edema localizado ou generalizado. Enfatizam ainda, a limpeza adequada da pele a fim de remover sujidade e prevenir a maceração, a frequência de limpeza necessita ser individualizada, uma vez que a limpeza excessiva provoca o ressecamento da pele e esta deve ser realizada através do uso de limpadores com PH entre 4,0 e 7,0. Como medidas preventivas a lesões por pressão relacionadas a dispositivos médicos trazem o rodízio de posicionamento e uso de coberturas de proteção como hidrocolóides e filme transparente.

Dentre todas as medidas elencadas a avaliação da pele, com ênfase nos pacientes mais suscetíveis a lesões por pressão, se mostra como a medida primária mais assertiva e que norteia as demais, além da conduta a ser tomada em cada caso.

Perrillat et al. (2020), sugere que fatores relacionados à patogenia do Covid-19 podem propiciar o maior risco de desenvolvimento de lesões por pressão em função da hipoxemia, lesão microvascular e trombose.

Nesse caminhar, tais aspectos inferem na necessidade de maiores estudos a fim de compreender a fisiopatologia específica deste perfil de paciente e viabilizar a correlação destes com um plano de cuidados de enfermagem preventivo e individualizado.

Perrillat et al. (2020), ainda sugere que o aumento de pacientes e a atuação de enfermeiras não especializadas em unidades de terapia intensiva levaram a um aumento da incidência na ocorrência de lesão por pressão. Esse relato nos traz a reflexão da importância de profissionais especializados e também aspectos relacionados ao subdimensionamento de pessoal, principalmente durante o enfrentamento à pandemia, a fim de prestar uma assistência com o menor risco possível ao dano.

Ramondetta et al. (2020), relata o caso de um paciente submetido a prona com o acometimento de lesão por pressão no mento e recomenda o uso de curativo com debridante enzimático e cobertura de espuma de poliuretano. Contribuindo com a reflexão inferida sobre as lesões por pressão, com o relato do caso acompanhado, e descrição do tratamento utilizado, sendo a espuma de poliuretano também descrita por outros autores como medida preventiva.

Dentre os estudos analisados encontramos similaridades identificadas pelos autores nas medidas de prevenção em quatro estudos sendo estes a avaliação diária da pele com identificação de riscos, limpeza adequada com solução de PH entre 4,0 e 7,0, 
controle de umidade, redistribuição de pressão com uso de coxins apropriados a cada caso, hidratação da pele, rotação da cabeça a cada duas horas, uso de cobertura protetora como hidrocolóide e filme transparente, acompanhamento por equipe multidisciplinar treinada e elaboração de protocolo clínico.

Martell e Orgil (2020) além de Perrillat et al. (2020) nos atentam ainda à observância e correção dos fatores intrínsecos envolvidos no desenvolvimento de lesões por pressão como aumento da liberação de occitocinas, disfunção endotelial, lesão microvascular, trombose, isquemia, hipoxemia, anemia e desnutrição.

Cabe-se ainda ressaltar os pontos, identificados pelos autores Busnardo et al. (2020) e Moore et al. (2020), como mais propícios ao desenvolvimento de lesões por pressão sendo estes as regiões do rosto (malar, nasal e frontal, mandibular e lábios), tórax, esternal, mama, clavículas, sínfise púbica, cristas ilíacas, pênis, planalto tibial, joelhos e dedos dos pés.

O uso de consulta virtual, mostrado por Martell e Orgil (2020), como medida inovadora de prevenção e direcionamento ao se determinar a melhor conduta terapêutica para o manejo de lesões por pressão, direcionada a especificidades de cada paciente, assim como a necessidade de profissionais capacitados na condução a este perfil de pacientes, exposto por Perrillat et al. (2020).

\section{Conclusão}

Dentre os achados resultantes desta pesquisa, nos chamou a atenção o uso da consulta virtual para auxílio na prevenção de feridas, entendemos esta menção como o uso da telemedicina para auxílio de especialista no traçar de conduta à escolha de cobertura e/ou intervenção mais apropriada. Os cuidados com a pele elencados por uma das autoras com ênfase na solução com PH entre 4,0 e 7,0 e avaliação diária também foi entendido como um achado digno de nota. Foi expressivo o entendimento da necessidade de profissionais especializados e/ou familiarizados com a rotina de cuidados ao paciente crítico como fator incisivo na qualidade dos serviços prestado e preventivo, uma vez que, entendemos que os conhecimentos dos pontos predispostos ao desenvolvimento de lesões por pressão devem deter atenção especial às medidas protetivas tão discursadas neste trabalho acadêmico. Cabe ainda menção à necessidade de uma visão holística no atendimento ao paciente acometido pela COVID-19, em posição prona, tendo em vista a imperatividade de sanar os fatores intrínsecos relacionados ao desenvolvimento de lesões por pressão. Este estudo encontrou como barreira ao seu desenvolvimento o baixo quantitativo de materiais disponíveis nos bancos de dados pesquisados, assim como seus baixos níveis de evidência científica. Entendemos este fenômeno dada a necessidade urgente de produção de conteúdo que vise subsidiar os profissionais atuantes na linha de frente do enfrentamento à pandemia por COVID-19.

Com base nos dados apresentados e dada a urgência pandêmica, sugere-se novas investigações no campo da aplicação de medidas preventivas tais como, avaliação diária da pele guiada por escalas específicas e sua associação com a condição clínica do paciente acometido por COVID-19, o uso de curativos e preventivos, aplicação de protocolos operacionais sistematizados bem como, estudos que observem os aspectos gerenciais como dimensionamento de pessoal, equipe especializada e treinamentos através do uso da simulação realística. A melhor observação desses pontos poderá ampliar o conhecimento acerca desse fenômeno e subsidiar melhores práticas em saúde.

\section{Referências}

Agência Nacional de Vigilância Sanitária-ANVISA. (2020). Orientações para serviços de saúde: medidas de prevenção e controle que devem ser adotadas durante a assistência aos casos suspeitos ou confirmados de infecção pelo novo coronavírus (SARS-CoV-2). http://portal.anvisa.gov.br/documents/33852/271858/Nota+Técnica+n+04-2020+GVIMS-GGTES-ANVISA/ab598660-3de4-4f14- 8e6f-b9341c196b28.

Botelho, L. L. R., de Almeida Cunha, C. C., \& Macedo, M. (2011). O método da revisão integrativa nos estudos organizacionais. Gestão e sociedade, 5(11), 121-136. http://www. gestaoesociedade.org/gestaoesociedade/article/view/1220/906

Busnardo, F. D. F., Monteiro, G. G., Mendes, R. R. D. S., Abbas, L., Pagotto, V. F., Camargo, C., ... \& Gemperli, R. (2020). A multidisciplinary approach to prevent and treat pressure sores in proned COVID-19 patients at a quaternary university hospital. Clinics, 75 . http://www.scielo.br/scielo.php?script=sci_arttext\&pid=S1807-59322020000100521\&lng=en. https://doi.org/10.6061/clinics/2020/e2196 
Campos, M. G. D. C. A., de Sousa, A. T. O., Vasconcelos, J. D. M. B., de Lucena, S. A. P., \& de Assis Gomes, S. K. (2016). FERIDAS COMPLEXAS E ESTOMIAS. João Pessoa: Ideia. http://www.corenpb.gov.br/wp-content/uploads/2016/11/E-book-coren-final-1.pdf

Durigon, E. L. (2020) Nota da Sociedade Brasileira de Microbiologia sobre o Novo Coronavírus nCoV - 2019. Sociedade Brasileira de Microbiologia - SBM. https://sbmicrobiologia.org.br/nota-da-sociedade-brasileira-de-microbiologia-sobre-o-novo-coronavirus-ncov-2019/

Martel, T., \& Orgill, D. P. (2020). Cutting Edge Care Delivery in Response to the Covid-19 Pandemic: Medical Device-Related Pressure Injuries During the COVID-19 Pandemic. Journal of Wound, Ostomy, and Continence Nursing, 47(5), 430. https://www.ncbi.nlm.nih.gov/pmc/articles/PMC7722289/

Moore, Z., Patton, D., Avsar, P., McEvoy, N. L., Curley, G., Budri, A., \& O'Connor, T. (2020). Prevention of pressure ulcers among individuals cared for in the prone position: lessons for the COVID-19 emergency. Journal of wound care, 29(6), 312-320. https://www.magonlinelibrary.com/doi/full/10.12968/jowc.2020.29.6.312

Moraes, J. T., Borges, E. L., Lisboa, C. R., Cordeiro, D. C. O., Rosa, E. G., \& Rocha, N. A. (2016). Conceito e classificação de lesão por pressão: atualização do National Pressure Ulcer Advisory Panel. Revista de Enfermagem do Centro-Oeste Mineiro, 6(2). http://seer.ufsj.edu.br/index.php/recom/article/view/1423

Morsch, A. L. B. C, Salvador, A. P., Garcez, S. D. F., Benati, R. M., \& Camera, F. D. (2018) Análise das repercussões clínicas da aplicação da posição prona em prematuros sem doença pulmonar. Perspectiva, Erechim. 42, 07-14 http://www.uricer.edu.br/site/pdfs/perspectiva/1005_654.pdf

NPIAP Painel. (2020) Dicas PIP de prevenção de lesões por pressão para posicionamento prono. National pressure injury advisory panel. https://npiap.com/page/COVID-19Resources

Perrillat, A., Foletti, J. M., Lacagne, A. S., Guyot, L., \& Graillon, N. (2020). Facial pressure ulcers in COVID-19 patients undergoing prone positioning: How to prevent an underestimated epidemic?. Journal of stomatology, oral and maxillofacial surgery, 121(4), 442-444. https://www.sciencedirect.com/science/article/abs/pii/S2468785520301543?via\%3Dihub

Ramondetta, A., Ribero, S., Costi, S., \& Dapavo, P. (2020). Pression-induced facial ulcers by prone position for COVID-19 mechanical ventilation. Dermatologic Therapy. https://www.ncbi.nlm.nih.gov/pmc/articles/PMC7300922/

Sanduzzi, A., \& Sanduzzi Zamparelli, S. (2020). Nasopharyngeal and oropharyngeal swabs, and/or serology for SARS COVID-19: what are we looking for?. International journal of environmental research and public health, 17(9), 3289.https://doi.org/10.3390/ijerph17093289

Soares, M. I., Resck, Z. M. R., Terra, F. D. S., \& Camelo, S. H. H. (2015). Sistematização da assistência de enfermagem: facilidades e desafios do enfermeiro na gerência da assistência. Escola Anna Nery, 19(1), 47-53. http://www.scielo.br/pdf/ean/v19n1/1414-8145-ean-19-01-0047.pdf

Souza, M. D., Silva, M. D., \& Carvalho, R. D. (2010). Revisão integrativa: o que é e como fazer. Einstein, 8 (1), 102-106. https://www.scielo.br/scielo.php?pid=S1679-45082010000100102\&script=sci_arttext\&tlng=pt

Ursi, E. S., \& Gavão, C. M. (2006). Prevenção de lesões de pele no perioperatório: revisão integrativa da literatura. Revista Latino-Americana de Enfermagem, 14(1), 124-131. http://www.scielo.br/scielo.php?script=sci_arttext\&pid=S0104-1692006000100017\&lng=en\&nrm=iso . 